\title{
Resultados da reabilitação neuropsicológica em paciente com doença de Alzheimer leve
}

Renata Ávila ${ }^{1}$

Recebido: 20/9/2002 Aceito:14/8/2003

\section{RESUMO}

Tradicionalmente sempre houve mais grupos de suporte e informação para familiares e cuidadores de pacientes com doença de Alzheimer (DA) do que para os próprios pacientes. Entretanto, com o aumento do número de diagnósticos de DA em sua fase inicial, esta realidade está se modificando. Cresce a demanda por tratamento tanto medicamentoso como comportamental para esses pacientes. O presente trabalho tem como objetivo descrever uma experiência de reabilitação neuropsicológica $(\mathrm{RN})$ em grupo, e posteriormente individual, com a mesma paciente com DA leve. CGP participou primeiramente de dois grupos de RN com duração de quatro meses cada, e em seguida iniciou RN individual por um período de 22 meses. Na RN foram trabalhados principalmente déficits de memória, linguagem e treinos de atividades de vida diária. Neste período de tratamento, verificou-se alteração positiva de seu escore no MEEM, além de nenhuma função cognitiva ter apresentado deterioração. Este estudo de caso corroborou pesquisas anteriores que apontam efeitos positivos da RN com pacientes com DA leve.

Unitermos: Reabilitação neuropsicológica, Doença de Alzheimer; Memória.

\section{ABSTRACT}

Results of neuropsychological rehabilitation in patient with mild Alzheimer's disease - A case study

Traditionally, Alzheimer's support and information groups have been developed for families and caregivers rather than for the actual patient. However, this reality is changing because of the increase in early AD diagnosis. The demand for medication and behavioral treatment for patients is also increasing. The purpose of this case report is to describe the neuropsychological rehabilitation (NR) of a mild AD patient in group and in individual sessions. First, CGP took part in two NR groups each for a 4 month period, and then started individual NR sessions for a 22 month period. In the sessions, memory and language abilities were the focus of the NR as well as the activities of daily living. During this period it was possible to observe positive changes in MMSE score, as will as preservation of other cognitive function scores. This single case study is in agreement of previous studies which also showed documented positive effects of NR with AD patients.

Keywords: Neuropsychological rehabilitation; Alzheimer's disease; Memory. 


\section{Introdução}

O envelhecimento populacional trouxe aumento da incidência de doenças neurodegenerativas e, entre elas, destaca-se a doença de Alzheimer (DA) que é o tipo de demência com maior incidência, sendo a mais estudada.

A reversão da deterioração causada pela DA ainda não foi descoberta e os tratamentos disponíveis atualmente visam à melhora cognitiva e diminuição de sintomas comportamentais. Isso é alcançado por meio de medicação e técnicas cognitivas de reabilitação, além de informações sobre a doença e o apoio a familiares e cuidadores.

A procura por esses tratamentos tem aumentado junto com o aumento do número de diagnósticos de DA. Assim, profissionais da área da saúde necessitam de treinamento especializado para tratar esses casos complexos, que, além de déficits cognitivos, alterações de comportamento e sintomas psiquiátricos, ainda apresentam questionamentos e problemas comumente verificados em idosos sem demência. Entre os mais comuns estão: discriminação social, mudança no papel social, solidão, problemas financeiros e desconforto emocional, associados ou não a algum comprometimento físico.

As alterações provocadas pela DA têm sido objeto de abordagem multidisciplinar e os tratamentos oferecidos são: medicação, reabilitação neuropsicológica (RN) e grupo informativo.

Em termos medicamentosos, os inibidores de acetilcolinesterase (AChE-I) estão entre os atuais tratamentos medicamentosos para a DA e têm apresentado resultados positivos, como melhora das funções cognitivas e do desempenho na realização das atividades de vida diária (AVD) desses pacientes (De Vreese et al., 2001). Por outro lado, intervenções não-medicamentosas, tais como a RN, também têm conseguido resultados positivos como melhora na cognição e nas AVDs, além de melhora na qualidade de vida (QV) dos pacientes (De Vreese et al., 2001).

A RN é um processo ativo que visa capacitar pessoas com déficits cognitivos causados por lesão ou doença, para que essas adquiram um bom nível de funcionamento social, físico e psíquico (Mc Lellan, 1991). Dessa maneira, a reabilitação implica maximizar funções cognitivas por meio do bem-estar psicológico, da habilidade em AVD e do relacionamento social (Clare e Woods, 2001). Além disso, também busca a diminuição dos déficits que ocasionam afastamento e isolamento social, dependência e discriminação (Kitwood, 1997).

A RN é um tratamento biopsicossocial que envolve os pacientes e seus familiares levando em conta as alterações físicas e cognitivas dos pacientes, o ambiente em que vivem, os fatores subjetivos e a sua biografia.
Déficits na memória são os primeiros sintomas de DA e são também os que causam maior frustração e incapacidade do paciente, comprometendo a realização de AVD e sua QV. Com isso, o paciente torna-se cada vez menos independente, aumentando a demanda por cuidados dos familiares e/ou cuidador.

A reabilitação de memória objetiva melhorar a performance do paciente por meio de técnicas específicas ou estratégias e auxílios externos. Há dois métodos de reabilitação da memória: 1 . a reabilitação que se fundamenta em trabalhar com a modalidade específica da memória que se encontra intacta, para compensar a modalidade que não está (Golstein e Beers, 1998); 2. a reabilitação que visa trabalhar as habilidades residuais da modalidade de memória que está deficitária, pois, qualquer que seja o prejuízo cognitivo, existe quase sempre a conservação de alguma capacidade funcional (Wilson, 1996).

Evidências da literatura mostram que o treino da memória em pacientes com DA leve a moderada produz resultados promissores, pois esse tipo de tratamento promove melhora da memória explícita e se estende para habilidades funcionais temporariamente. Assim, De Vreese et al. (1998) compararam 24 pacientes com DA pareados em relação à escolaridade e à gravidade da doença, divididos em quatro grupos; placebo, tratamento medicamentoso com AChE-I, treinamento cognitivo (TC) e tratamento combinado (TC + AChEI) e os resultados mostraram que os pacientes que receberam o tratamento combinado apresentaram melhor efeito terapêutico em relação ao funcionamento cognitivo e performance em AVD.

Bolognani et al. (1999) avaliaram os efeitos da RN em pacientes com demência em nove indivíduos (sete com DA e dois com demência vascular) que foram atendidos em dois grupos, semanalmente, por seis meses. Os resultados indicaram estabilidade, ou um pequeno declínio, em algumas das escalas e testes reaplicados após seis meses de acompanhamento, mas a melhora no escore médio do Miniexame do Estado Mental (MEEM) revelou um tamanho de efeito de 0,46 , indicando um efeito positivo do treinamento cognitivo realizado, já que a medicação desses pacientes não foi alterada.

Clare (2001) utilizou o método da aprendizagem sem erro, bem como auxílios externos, para treinar pacientes com DA leve. O treino consistia na aprendizagem de nomes e informações. Foi observada melhora na memória de pacientes, permanecendo assim por até seis meses após o término dos treinos.

Os resultados preliminares de Bottino et al. (2002) em ensaio clínico de um grupo de seis pacientes com DA leve por sete meses mostrou que, ao final do acom- 
panhamento, houve estabilização ou discreta melhora dos déficits cognitivos e das AVD dos pacientes, concluindo que o tratamento combinado pode auxiliar na estabilização ou na redução dos déficits.

Farina et al. (2002) compararam dois tratamentos em que um estimulava a memória procedural com treino em AVD e o outro realizava treinos específicos de função cognitiva como memória, atenção e linguagem, e concluíram que ambos os grupos apresentaram melhora na performance em AVD, embora as teses neuropsicológicas tivessem sugerido que o treino de AVD é mais eficaz que a estimulação das funções cognitivas deficitárias.

Pesquisa realizada recentemente por nosso grupo selecionou seis pacientes com DA leve a moderada para realizar tratamento combinado por um período de quatro meses, com sessões duas vezes por semana. Ao final do tratamento houve estabilização ou discreta melhora dos déficits cognitivos com aumento do MEEM de 22,20 para 23,80 pontos e de AVD, além de diminuição de sintomas psiquiátricos dos pacientes (Ávila et al., 2002).

Foi também demonstrada nos últimos anos a eficiência de certas técnicas de memória, entre elas:

1. as que se baseiam em trabalhar memória implícita, geralmente intacta, como, por exemplo, utilizar gestos, emoção, dar opinião pessoal sobre determinado assunto;

2. compensação dos déficits de memória explícita por meio de treino do uso de auxílios externos, como agenda, computador, pager, bloco de recados, etc.

3. facilitação da memória explícita residual, por meio de suportes estruturados, tanto de codificação como de resgate. Este trabalho é realizado fornecendo-se as mesmas pistas durante a codificação da informação quando no resgate.

Logo após a fase amnéstica de DA, é comum haver declínio da linguagem, principalmente nomeação, definição semântica, bem como de funções executivas (Perry e Hodges, 1999). Sendo assim, em pacientes com DA inicial, a RN não visa melhorar apenas a memória, mas também as funções antes mencionadas. Entretanto, ainda não há estudos específicos de RN com pacientes com DA envolvendo tais funções.

O objetivo deste estudo de caso é descrever o trabalho de $\mathrm{RN}$ em grupo e individual realizado com paciente com DA leve e avaliar os benefícios deste tratamento na memória e nas outras funções cognitivas. Inicialmente, a paciente foi tratada exclusivamente com medicamentos por 3 meses, e posteriormente foi associado ao medicamento o grupo de $\mathrm{RN}$, que durou 4 meses. Depois a paciente ficou 8 meses sem treinamento cognitivo e posteriormente participou de novo grupo de $\mathrm{RN}$ por mais 4 meses. Ao término deste segundo grupo, ficou 2 meses sem RN, iniciando então atendimento individual com duração de 22 meses. Neste período total de acompanhamento ( 3 anos e 9 meses) o tratamento medicamentoso não foi interrompido nem alterado.

\section{Método}

\section{Caso}

CGP, hoje com 73 anos, sexo feminino, viúva e aposentada há mais de 12 anos. Ela vive com uma cuidadora, tem nível superior completo e trabalhou muitos anos como secretária bilíngüe, além de ter sido professora de piano. A paciente tem duas filhas casadas e dois netos.

Seu primeiro atendimento na clínica de memória do Projeto Terceira Idade (PROTER) do Hospital das Clínicas da Universidade de São Paulo foi em março de 1999. Ela chegou ao hospital com queixa de esquecimentos freqüentes que vinham ocorrendo havia dois anos, além de sintomas depressivos sem remissão, mesmo após medicação. CGP foi diagnosticada com DA inicial, após ter realizado exames de sangue, EEG, RM e avaliação neuropsicológica. Iniciou tratamento com rivastigmina $6,0 \mathrm{mg}$ e risperidona $1,0 \mathrm{mg}$. O uso da risperidona deu-se em decorrência de agitação noturna.

Iniciou grupo de $\mathrm{RN}$ no segundo semestre do ano de 1999. Esse grupo era formado por seis pacientes com DA leve e teve duração de 4 meses, com sessões semanais de 90 minutos. No segundo semestre de 2000, participou novamente de grupo $\mathrm{RN}$, nos mesmos moldes do anterior.

Paralelamante ao grupo de pacientes, havia o grupo informativo e de apoio para familiares e cuidadores.

Esses grupos foram realizados no PROTER. As sessões com os pacientes foram conduzidas por uma neuropsicóloga e uma fonoaudióloga, e os grupos com os familiares por uma psicóloga e uma psiquiatra. As avaliações foram realizadas por profissionais (psiquiatra e psicóloga) independentes do grupo de terapeutas envolvidos com o trabalho de reabilitação.

Em março de 2001, CGP iniciou RN individual em sua casa com uma neuropsicóloga, constando de sessões semanais com duração de 1 hora cada, e as informações eram passadas para a cuidadora e para os familiares sempre que necessário. 
O objetivo dos dois grupos de $\mathrm{RN}$ nos quais CGP participou foi o de trabalhar aspectos relacionados aos principais prejuízos cognitivos verificados na DA leve: a memória e a linguagem. Este trabalho foi realizado por meio de mecanismos compensatórios e de treinos específicos para melhorar a performance de algumas funções cognitivas, objetivando minimizar dificuldades em AVD e, dessa maneira, melhorar também a QV desses pacientes.

\section{Avaliação}

Os treinamentos de RN possuem avaliação antes e após o tratamento que serão aqui comparadas. A avaliação de eficácia foi feita por meio do Miniexame do Estado Mental (MEEM) (Burns et al., 1999), e a bateria neuropsicológica foi formada pelos seguintes testes: cubos, dígitos e vocabulário (WAIS-R) (Wechsler, 1999); Boston Naming Test (Spreene, Strauss, 1998); leitura, escrita e compreensão; Fuld Object Memory Evaluation (FOME) (Lesak, 1995); Trail Making Test A e B (Wechsler, 1999); fluência verbal categoria animal (Spreen e Strauss, 1998), desenho do relógio (Strub e Black, 1988) e cubo de Necker (Strub e Black, 1988).

\section{Reabilitação neuropsicológica}

Os dois atendimentos em grupo foram estruturados e planejados da mesma maneira. As sessões sempre iniciavam com atividades de orientação temporoespacial e treino dos nomes das pessoas do grupo. As atividades eram divididas em: treino de funções cognitivas abrangendo atenção, linguagem e memória (implícita e explícita), exercícios de socialização em que os pacientes dividiam experiência e fatos significativos de suas vidas, além de treinos de AVD. Entre eles: anotar e transmitir um recado, dar e receber um telefonema, e ler uma reportagem no jornal ou revista.

Exercícios que visavam ampliar a capacidade de manter a atenção em um ou mais estímulos foram feitos em associação a exercícios específicos de linguagem como resgate verbal, fluência verbal, manutenção do discurso, fala espontânea e escrita. Esses treinos também visavam trabalhar a memória semântica e episódica.

Para trabalhar a memória recente, eram feitos treinos de associações verbais e visoverbais, que facilitam tanto a codificação como o posterior resgate. É importante fornecer as mesmas pistas para o paciente nesses dois momentos.

$\mathrm{O}$ atendimento individual teve duração de 22 meses. Neste período foram realizados treinos de uso de auxílios externos, como usar agenda e calendário e bloco para anotar recados. Foram trabalhadas atividades que usam memória implícita, já que esta permanece relativamente intacta até a fase grave da doença, com o uso do computador. Para tanto, todos os passos foram anotados e fixados ao lado do computador, e as principais teclas foram numeradas e suas funções foram explicadas em uma legenda, também fixada ao lado dele. É importante ressaltar que CGP datilografava na época em que trabalhava, assim, essa tarefa tornou-se menos complexa para ela, conseguindo ainda hoje digitar com rapidez e sem erros.

A memória remota, que também está praticamente preservada na DA leve, também foi trabalhada, já que a reminiscência é uma atividade prazerosa, em que o diálogo fica sem quebras, pois o paciente recorda bem de fases anteriores de sua vida. Com esta paciente foi iniciado um livro autobiográfico, que contém todas as etapas de sua vida e os principais acontecimentos.

Além da memória, também foram treinadas outras funções cognitivas, como função executiva e linguagem. Para o treino de atenção foram utilizados muitos estímulos visuais, em que a paciente tinha que descrever com detalhes certas figuras, ou encontrar determinados objetos pertencentes a uma categoria específica ou começando com determinada letra. Esses exercícios também trabalhavam nomeação e fluência verbal. Treinos de nomeação foram feitos com objetos da vida diária da paciente, e com jogos em que eram dadas pistas para ela acertar que objeto estava sendo descrito. As pistas eram lidas até a paciente dizer que já sabia o que era.

Para a memória de trabalho foram realizados jogos tanto com o computador como com lápis e papel, em que a paciente tinha que formar o máximo de palavras que conseguisse com as letras dadas. Além disso, foram feitos exercícios de cálculos, sempre relacionados com situações de vida diária, como pagar uma conta, receber e conferir o troco.

Outra atividade muito realizada foi a de leitura e compreensão de textos curtos, e de pequenas crônicas, em que sua capacidade de abstração também foi trabalhada. Foram feitas leituras em português e inglês, já que a paciente referia seu medo de esquecer o que sabia de inglês.

Todos os treinos realizados se basearam no princípio da "aprendizagem sem erro", em que era dito e freqüentemente relembrado à paciente que era proibido adivinhar quando tinha dúvidas e que deveria pedir pistas até saber a resposta correta. À medida que a paciente ia acertando, o número de pistas ia sendo diminuído.

Além do treino das principais funções cognitivas prejudicadas em função da DA, e com o aprimoramento das funções não prejudicadas, também foram traba- 
lhadas queixas e inseguranças da paciente, em que geralmente se enfocava a necessidade de ela valorizar suas habilidades preservadas e tudo que ainda poderia fazer, aprender e aproveitar na vida. Além disso, atividades externas de lazer e de interesse da paciente eram feitas a cada dois meses. Para tanto, a psicóloga e a paciente procuravam no jornal o que havia na cidade, eram selecionadas algumas opções e a paciente escolhia o que mais lhe interessava. Ao final de cada atividade externa era solicitado que a paciente escrevesse sua impressão sobre o passeio.

\section{Resultados}

Os escores detalhados do MEEM feitos nas 6 avaliações podem ser vistos na gráfico 1 . A primeira, feita em junho de 1999, três meses após ter iniciado o tratamento medicamentoso com AChE-I e antes do início do grupo de RN, CGP obteve escore 23. Após 4 meses de reabilitação, a paciente obteve escore de 22. Em junho de 2000, foi realizada outra avaliação antes do início do segundo grupo de RN. CGP obteve escore 22, mantendo-se estável desde a última avaliação, isto é, por 6 meses. Ao final do grupo de $\mathrm{RN}$, a paciente foi novamente reavaliada e seu escore no MEEM foi 23.

CGP permaneceu dois meses sem treinamento cognitivo e, em março de 2001, iniciou RN individual e, após dez meses, foi novamente avaliada. Nessa avaliação, janeiro de 2002, seu escore passou para 25, permanecendo inalterado por um ano, já que obteve o mesmo escore na última avaliação, em janeiro de 2003. Ou seja, apresentando um aumento de dois pontos da última avaliação feita, antes do tratamento individual iniciar, e um aumento de 3 pontos, se comparado com o escore do primeiro MEEM.

Sabe-se que a queda média do escore no MEEM de pacientes com DA é de quase três pontos por ano (Morris, 1994; Salmon et al., 1990), o que confirma, portanto, os benefícios de seu tratamento. Além disso, nas avaliações de 2002 e 2003, mostrou-se capaz de relembrar um objeto no item evocação. Isso nos dá indícios de melhora em sua capacidade de armazenamento e posterior resgate do material verbal.

Cumpre lembrar que este item de memória e os itens de orientação temporal são os que primeiro apresentam prejuízos na DA, sendo os mais difíceis para recuperação sem ajuda de pistas ou auxílios. Este dado ainda apresenta maior valor quando comparado aos resultados obtidos nos testes neuropsicológicos, que serão discutidos a seguir.

Os detalhes da avaliação neuropsicológica estão resumidos no gráfico 2. A avaliação inicial foi realizada em junho de 1999, e a última em janeiro de 2003. Analisando sua performance durante todo este tempo, podese inferir que CGP teve progressos, e mais que isto, não apresentou a deterioração esperada na maioria das funções cognitivas.

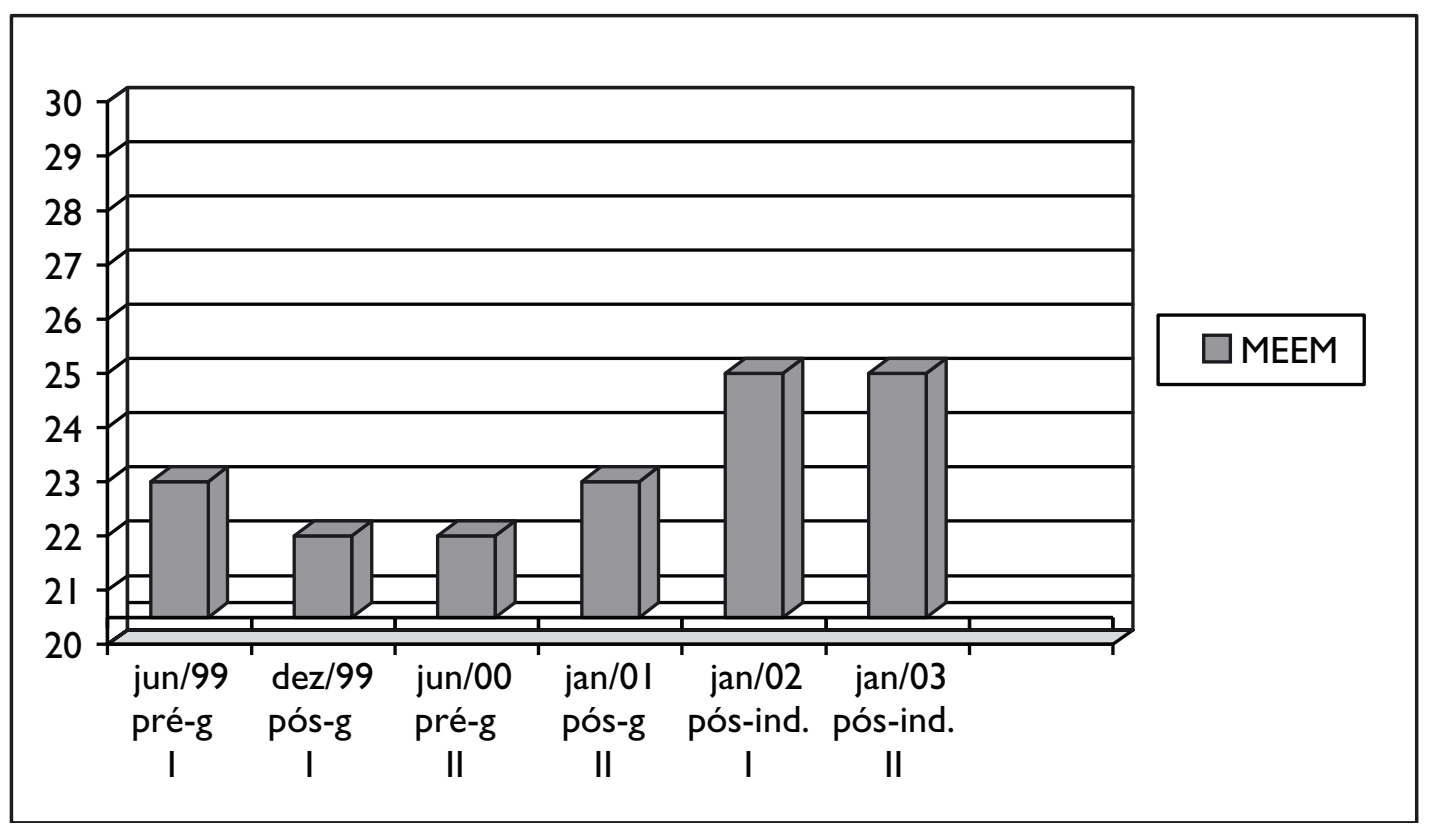

Pré-g: pré-reabilitação em grupo; pós-g: pós-reabilitação em grupo; pós-ind.: pós-reabilitação individual.

Gráfico 1 - Resultados do MEEM antes e após o tratamento. 


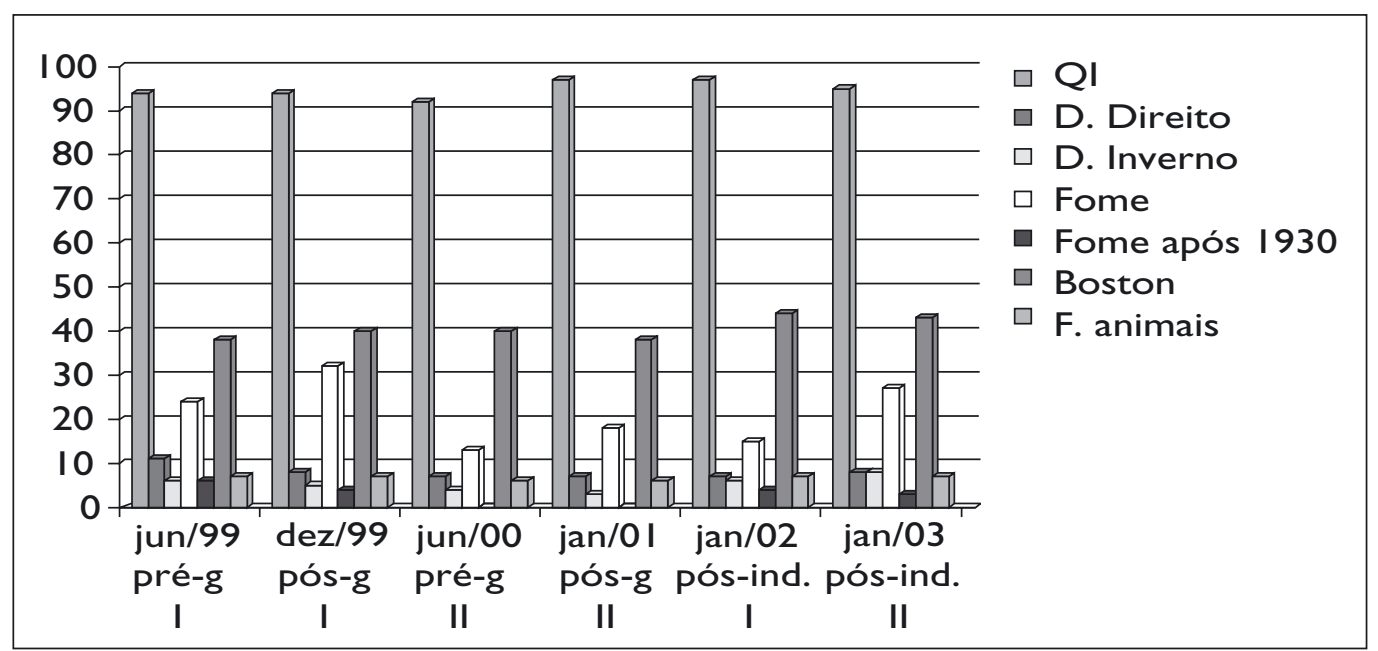

Os resultados do gráfico são os pontos brutos obtidos nos testes; QI: quociente intelectual; D. Direto: dígitos diretos; D. Inverso: dígitos inversos; FOME: Fuld Object Memory Evaluation; Boston: Boston Naming Test; F. animais: fluência verbal, categoria semântica - animais; pré-g: pré-reabilitação em grupo; pós-g: pós-reabilitação em grupo; pós-ind.: pós-reabilitação individual.

Gráfico 2 - Resultados das avaliações neuropsicológicas.

Por meio dos subtestes da escala WAIS-R, vocabulário e cubos, foi calculado o QI estimado da paciente, e verificou-se que não houve quase alteração no QI no decorrer destes anos, só pequenas oscilações nos valores brutos dos subtestes.

Analisando os testes de atenção, sua amplitude atencional (dígitos direto) teve uma queda mesmo participando de grupo de $\mathrm{RN}$, e outra queda após uma pausa da reabilitação no primeiro semestre de 2000 . Sua capacidade de controle mental (dígitos inverso) teve oscilações em 3 anos, voltando para o mesmo resultado obtido em 1999. Desta maneira, não houve deterioração neste período. Em atividade que requer rastreamento visual e atenção para um estímulo (Trail Making A), não apresentou melhora após participar do primeiro grupo de RN, mas após o segundo grupo teve melhora significativa, mantendo o mesmo resultado estável até 2003. Entretanto, quando a atividade demandava atenção dividida (Trail Making B), apesar de ter melhorado seu desempenho entre os anos de 1999 e 2001, período este que participou dos dois grupos, após ter iniciado as sessões individuais piorou, voltando a ter o mesmo resultado do início do tratamento.

Esse dado nos faz pensar que as atividades e discussões em grupo são positivas para trabalhar a atenção dividida desses pacientes, o que não ocorre em trabalhos individuais. Além disso, nesses meses de reabilitação individual, pouca atenção foi dada aos déficits de atenção dividida, sendo mais trabalhados a amplitude atencional e o rastreamento para um estímulo só.

No teste de memória (FOME) em que a paciente primeiramente reconhece o objeto pelo tato, depois visualmente e por último faz evocação verbal, CGP obteve resultado surpreendente. Nesse teste, são realizadas cinco evocações, em que provas de interferência são intercaladas (fluência verbal), e depois de 30 minutos são feitas novamente evocação e prova de reconhecimento. Seu resultado no número total de objetos evocados aumentou neste último ano, significando que tem capacidade de aprendizagem e que esta pode ser otimizada com treinos. Além disso, também apresentou melhora na evocação dos objetos após tarefa de interferência, o que indica que sua capacidade de armazenamento e posterior evocação melhorou. Este dado corrobora o resultado obtido no MEEM. É importante ressaltar que pacientes com DA apresentam quedas significativas de desempenho deste teste no decorrer dos anos, principalmente na evocação tardia após interferência. Para esse tipo de atividade, o atendimento individual mostrou-se mais eficaz, em que treinos mais específicos e intensos puderam ser feitos, com técnicas de associações para facilitar o armazenamento e evocação.

Em testes que avaliam praxia construtiva (relógio e cubo de Necker), após ter iniciado RN em grupo, obteve melhora, chegando ao desempenho classificado como normal para as duas tarefas, mantendo-se estável até hoje. Este resultado surpreende principalmente no desenho tridimensional, pois ao logo da doença se observa aumento do prejuízo nesta função cognitiva.

Em provas de linguagem, escrita e compreensão, teve resultados normais até o último semestre de 2000, em que apresentou leitura sem pontuação e escrita com erros. Após iniciar reabilitação individual, seus resultados voltaram para a faixa da normalidade. Novamente, podemos pensar que treinos de leitura principalmente 
são feitos de maneira mais eficaz em atendimentos individuais, em que o paciente pode ser corrigido e observado com maior freqüência.

$\mathrm{O}$ teste de Boston, que avalia capacidade de nomeação, foi o que apresentou resultado mais surpreendente, com melhora gradativa no decorrer desses anos. E com relação à capacidade de nomeação, pode-se inferir que é decorrente da atenção especial que foi dada a este aspecto da linguagem, principalmente neste último ano de reabilitação.

Em atividades que avaliam funções motoras, apresentou resultado estável em 3,5 anos, assim como suas habilidades para cálculos simples. Já para testes que medem fluência verbal para categorias semânticas, apresentou desempenho estabilizado e oscilações no número de palavras ditas. Já para a categoria animais, seu desempenho manteve-se estável.

\section{Discussão}

Algumas limitações metodológicas devem ser mencionadas, tais como tratar-se de relato de um único caso, portanto sem uma comparação com um pacientecontrole e submetido à reabilitação, o que permitiria avaliar com maior precisão o efeito da medicação isoladamente, ou associada à reabilitação. Entretanto, é possível argumentar que a melhora dos déficits cognitivos e das AVD observada nos pacientes tratados com rivastigmina ocorreria até o terceiro mês de tratamento medicamentoso, havendo depois estabilização ou até uma discreta piora (Rosler et al., 1999). O uso da risperidona é indicado para alterações de comportamento em pacientes com DA e, quando há evidências na literatura que mostram que seu uso não beneficia a cognição dos pacientes, age somente sobre o comportamento (Weiser et al, 2002; Rainer et al., 2001; Laks et al., 2001).

Como a paciente estudada já estava usando rivastigmina e risperidona há três meses antes do início da $\mathrm{RN}$, é possível argumentar que os efeitos da medicação já teriam ocorrido, o que representa argumento favorável ao efeito benéfico da $\mathrm{RN}$.

Outro fator que não deve ser desprezado é o possível efeito benéfico dos atendimentos intensivos tanto em grupo quanto individual prestados a paciente $\mathrm{e}$ seus familiares, o que pode ter contribuído também para melhora em seu desempenho cognitivo e funcional, além de sintomas psiquiátricos.

\section{Conclusão}

A RN aplicada em grupo pequeno e homogêneo, assim como a individual, mostrou efeitos positivos. Os dados indicam que, com o trabalho de $\mathrm{RN}$ associada à AChE-I, o paciente é capaz de manter o paciente com menos déficits, melhorando sua independência e QV. Além disso, é capaz de elevar seu escore mental e algumas funções cognitivas, fazendo supor que seu potencial de habilidades preservadas pode ser ativado com esse tipo de intervenção.

Dessa maneira, quanto mais cedo for feito o diagnóstico de DA e mais cedo o tratamento for iniciado, melhores resultados serão obtidos, pois o paciente poderá manter-se em um nível cognitivo e funcional mais elevado. Isso possibilitará ainda que o paciente permaneça mais tempo independente, evitando inclusive maiores sobrecargas a seus familiares.

Pequenas alterações cognitivas ou até mesmo a estabilização de algumas funções cognitivas já revelam na realidade um grande ganho, no caso de pacientes com doença neurodegenerativa. Além disso, muitas vezes, pequenas alterações nas escalas e testes podem representar grande benefício na vida dos pacientes.

\section{Referências bibliográficas}

Ávila, R.; Mıоттo, E.; Carvalho, I. et al. - Neuropsychological rehabilitation of memory deficits in patients with Alzheimer's disease - artigo não publicado.

Bottino, C.M.C.; Carvalho, I.; Alvarez, A.M.; Ávila, R. et al. Reabilitação cognitiva em pacientes com doença de Alzheimer: relato de trabalho em equipe multidisciplinar. Arq Neuropsiquiatr 60 (1): 70-9, 2002.

Bolognani, S.A.P.; Fabricio, A.M.; Garcia, J.L.; Cid, C.G.; Faria, G.C. Nomura, S.; Camargo, C.H.P.; Bottino, C.M.C. - Neuropsychological Rehabilitation in a group of patients with mild dementia: preliminary results. Neurobiology of Aging 19: 101(A425), 1999.

Burns, A; Lawlor; B; Craig, S. - Assessment scales in old age. Martin Dunitz, London, 1999.

Clare, L.; Woods, R.T. - Cognitive rehabilitation in dementia. A special issue of neuropsychological rehabilitation. Psychological Press New York, 2001.

DeVreese, L.P.; Iacono, S.; Finelli, C. et al. - Enhancement of therapeutic effects of drug treatment in DAT when combined with cognitive training? Preliminary results of a three-month program. Neurobiology of Aging 19 (S4): S213, A893, 1998.

De VReese, L.P.; Neri, M.; Fioravanti, M.; Belloi, L.; Zanetti, O. - Memory Rehabilitation in Alzheimer's disease: a review of progress. Int. Journal of Geriatric Psychiatry 16: 794-809, 2001.

Farina, E.; Fioravanti, R.; Chavari, L; Imbornone, E. et al. - Comparing two program of cognitive training in Alzheimer's disease: a pilot study. Acta Neurol Scand 105: 365-71, 2002.

Goldstein, G.; Beers, S. - Assessment and planning for memory retraining. In: Golstein G, Beeres S.: Rehabilitation. Plenum, New York, pp. 229-43, 1998.

Kitwood, T. - Dementia reconsidered: the person comes first. Open University, Buckingham, 1997.

Laks, J.; Engelhardt, E.; Marinho, V. et al. - Efficacy and safety of risperidone oral solution in agitation associated with dementia in the elderly. Arq Neuropsiquiatr 59 (4): 859-64, 2001. 
Lezak, M. - Neuropsychological Assessment. 3 ed. Oxford University, New York, 1995.

Mc Lellan, D.L. - Functional recovery and principles of disability medicine. In: Swash M.; Oxbury J. Clinical Neurology. Churchill Livingstone, London, pp. 768-90, 1991.

Morris, J.C. et al. - The consortium to establish a registry for Alzheimer's disease (CERAD). Part IV. Rates of cognitive change in the longitudinal assessment of probable Alzheimer's disease. Neurology 44: 1215-20, 1994.

Perry, R.J.; Hodges, J.R. - Attention and executive deficits in Alzheimer's disease - a critical review. Brain 122: 383-404, 1999.

Rainer, M.K.; Masching, A.J.; ArtL, M.G.; KraXberger E.; Haushofer M. - Effect of risperidone on behavioral and psychological and cognitive function in dementia. J Clin Psychiatry 62 (11): 894-900, 2001.

Rosler, M.; Anand, R.; Cicin-Sain, A et al. - Efficacy and safety of rivastigmine in patients with Alzheimer's disease: international randomized controlled trail. BMJ 318: 633-8, 1999.
Salmon, D.P.; Thal, L.J.; Butters N.; Heindel, W.C. - Longitudinal evaluation of dementia of the Alzheimer type: comparison of three standardised mental state examinations. Neurology 40: 1225-30, 1990 .

Spreen, O.; Strauss, E. - A Compendium of Neuropsychological Tests- Administration, Norms and Commentary. 2.ed. Oxford University, New York, 1998.

Strub, R.L.; Black, F.W. - The mental states examination in neurology. 2 ed. Davis Company, Philadelfia, 1988.

Wechsler, D. - Wechsler Intelligence Scale Revised. Psychological, New York, 1999.

Weiser, M.; Rotmensch, HH; Korzyn, A.D. et al. - A pilot, randomized, open-label trial assessing safety and pharmakokinetic parameters of co-administration of rivastigmina with risperidone in dementia patients with behavioral disturbances. Int $\mathrm{J}$ Geriatric Psychiatric 17 (4): 343-6, 2002.

WiLson, B.A. - Reabilitação das deficiências cognitivas. In: Notrini, R.; Caramelli, P.; Mansur, L.L.: Neuropsicologia das bases anatômicas à reabilitação. Clínica neurológica, São Paulo, HCFMUSP, pp. 314-343, 1996. 